\title{
Erratum to: Accumulation of fatty acids in Chlorella vulgaris under heterotrophic conditions in relation to activity of acetyl-CoA carboxylase, temperature, and co-immobilization with Azospirillum brasilense
}

\author{
Luis A. Leyva • Yoav Bashan • Alberto Mendoza •
}

Luz E. de-Bashan

Published online: 21 October 2014

(C) Springer-Verlag Berlin Heidelberg 2014

Erratum to: Naturwissenschaften (2014) 101:819-830

DOI 10.1007/s00114-014-1223-x

The original publication of this article contains a mistake in the article title. Instead of

"Accumulation fatty acids of in Chlorella vulgaris under heterotrophic conditions in relation to activity of acetyl-CoA carboxylase, temperature, and co-immobilization with Azospirillum brasilense"

it should have been

"Accumulation of fatty acids in Chlorella vulgaris under heterotrophic conditions in relation to activity of acetyl-CoA carboxylase, temperature, and co-immobilization with Azospirillum brasilense".

The online version of the original article can be found at http://dx.doi. org/10.1007/s00114-014-1223-x.

L. A. Leyva · Y. Bashan • L. E. de-Bashan $(\bowtie)$

Environmental Microbiology Group, Northwestern Center for

Biological Research (CIBNOR), Av. IPN \#195, La Paz,

B.C.S. 23096, Mexico

e-mail: luzb@cals.arizona.edu

L. E. de-Bashan

e-mail: legonza104@cibnor.mx

Y. Bashan • L. E. de-Bashan

The Bashan Foundation, 3740 NW Harrison Blvd., Corvallis,

OR 97330, USA

A. Mendoza

Centro de Biotecnología Genómica, Instituto Politécnico Nacional,

Blvd. Del Maestro s/n, Col. N. Mendoza, Reynosa,

Tamaulipas 88710, Mexico 